\title{
Stereoscopic Space Map - Semi-immersive Configuration of 3D- stereoscopic Tours in Multi-display Environments
}

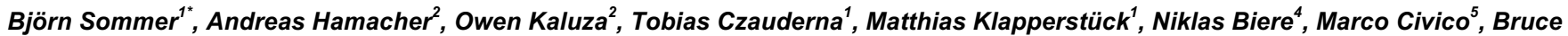 \\ Thomas $^{6}$, David G. Barnes ${ }^{2,3}$, Falk Schreiber ${ }^{1}$ \\ ${ }^{1}$ Faculty of Information Technology, Monash University, 3800 Clayton, Australia \\ ${ }^{2}$ eResearch Centre, Monash University, 3800 Clayton, Australia \\ ${ }^{3}$ Monash Biomedical Imaging, Monash University, Clayton, Australia \\ ${ }^{4}$ Experimental Biophysics \& Applied Nanosciences, Bielefeld University, 33615 Bielefeld, Germany \\ ${ }^{5}$ Gymnasium Schloss Holte-Stukenbrock, 33758 Schloß Holte-Stukenbrock, Germany \\ ${ }^{6}$ School of Computer and Information Science, University of South Australia, 5095 Mawson Lakes, Australia \\ *Corresponding author: bjoern@CELLmicrocosmos.org
}

\begin{abstract}
Although large-scale stereoscopic $3 D$ environments like CAVEs are a favorable location for group presentations, the perspective projection and stereoscopic optimization usually follows a navigator-centric approach. Therefore, these presentations are usually accompanied by strong side-effects, such as motion sickness which is often caused by a disturbed stereoscopic vision. The reason is that the stereoscopic visualization is usually optimized for the only head-tracked person in the CAVE - the navigator - ignoring the needs of the real target group - the audience.
\end{abstract}

To overcome this misconception, this work proposes an alternative to the head tracking-based stereoscopic effect optimization. By using an interactive virtual overview map in $3 D$, the pre-tour and on-tour configuration of the stereoscopic effect is provided, partly utilizing our previously published interactive projection plane approach. This Stereoscopic Space Map is visualized by the zSpace $200 \AA$, whereas the virtual world is shown on a panoramic $330^{\circ} \mathrm{CAVE2} 2^{T M}$.

A pilot expert study with eight participants was conducted using pre-configured tours through $3 D$ models. The comparison of the manual and automatic stereoscopic adjustment showed that the proposed approach is an appropriate alternative to the nowadays commonly used head tracking-based stereoscopic adjustment.

\section{Introduction}

In the last two decades, CAVEs (CAVE Automatic Virtual Environments) were used for a wide range of applications. Traditional CAVEs - such as the one developed by Cruz-Neira et al. in the $1990 \mathrm{~s}$ - consist of at least three display components, representing the front, side, top and/or bottom perspective [1]. A well-known alternative are HMDs (head-mounted displays). Coming a long way since 1968 [2], HMDs - such as the Oculus Rift - might finally become in 2016 the future standard in many VR-relevant application areas, although the visual acuity and binocular resolution of CAVEs are usually significantly higher than the one of HMDs [3]. The major advantage is the mobility, the easy set-up of these systems and the low acquisition costs. It can be predicted that many application areas which were formerly reserved for expensive CAVE-related display setups in the past will be soon completely covered by these HMDs. Therefore, the research and development of large display environments have to be focused now on their specific application areas.

\section{Stereoscopic Visualization in CAVEs}

A major drawback of CAVEs is that - although they are huge enough to be used by multiple persons - it is only possible to optimize the Stereoscopic $3 D$ ( $S 3 D$ ) visualization for a single head-tracked person (in case the visualization of the virtual world should be consistent over all perspectives). But a large advantage in comparison to HMDs is the fact that the virtual world (which is the digital world the user should be immersed in), the real world (which is the world we are living in), as well as the people inside the CAVE are visible. Therefore, they are optimal for group presentations and should also be optimized for this kind of presentations.

As previously mentioned, the stereoscopic visualization in CAVEs is usually based on a tracked pair of glasses worn by the navigator - the person who navigates through a virtual environment during the presentation. In this way, the position of the tracked pair of glasses can be used as a reference point to compute the eye distance based on the distance between the tracked glasses and the closest object in the virtual world. As long as the audience - formed by the passive attendees of a presentation - is physically located close to the navigator and the navigator is not physically moving, this approach might be sufficient at first glance. But moreover, the projection matrices are recomputed based on the tracked pair of glasses so that the perspective projection depends on the point of view of the navigator. Therefore, a) the perspective projection and b) the stereoscopic adjustment is distorted for the audience, and c) if the navigator starts to physically move, also the virtual environment seems to be in motion from the perspective of the audience.

Of course, especially in large CAVEs, the audience is usually separated from the navigator, because the navigator is usually also the presenter, communicating with the audience while navigating with a wand-like device.

Summing up it can be stated that the use of head-tracking in group presentation should be prevented in CAVEs. An alternative method is required to optimize the stereoscopic effect to prevent strong side-effects. It is known that a bad configuration of the eye distance can cause eye strain, head aches, vertigo, or even motion sickness, also known as cyber sickness [4]-[8]. Especially the last mentioned problem occurs quite often in virtual environments such as CAVEs. 


\section{Pre-tour and on-tour stereoscopic configuration}

There are various ways to tackle and analyze these visual problems, e.g. for stereoscopic motion pictures [9]-[11]. Previously we have introduced an approach to optimize the stereoscopic setting in interactive environments - the interactive projection plane $S 3 D$ method [12]. The introduced methodology is especially relevant in case virtual environments with huge differences in scale have to be explored, such as biological cell models with scale variations of up to a factor of 100,000 . For example, at the mesoscopic level, a ribosome has a size of $23 \mathrm{~nm}$, a wall of a plant cell might have a size of approx. $15,000 \mathrm{~nm}$. At the molecular level, a smaller molecule might have a size of around $0.1 \mathrm{~nm}$. These differences in scale do usually not have to be taken into account for regular virtual environments, as it is not required to move very close to extremely small objects. Therefore, in regular virtual worlds, like for example architectural models, the near clipping plane of the virtual camera usually prevents that objects can move very close to the camera.

We therefore present here a new method to optimize the stereoscopic effect

- $\quad$ pre-tour, i.e., during the preparation of a tour without an

audience, and

- on-tour, i.e., during the presentation in front of an audience.

Given a set of 3D models - in our case a number of biological cell models - these models should be presented to an audience. A related real life scenario would be the exploration of different cell components by the navigator with the purpose to explain their location, shape and functionality to an audience inside a CAVE. Based on the cell model, a tour has to be prepared. The tour consists of a number of tour points and their sequential order. Each tour point contains information about a) the camera position, b) the camera orientation, and c) the stereoscopic eye distance at this certain position. During the configuration of the tour, no audience is present and the navigator can prepare the tour. We call this the pre-tour situation.

During the on-tour situation, the navigator is presenting the preconfigured tour to an audience. Moreover, it might occur that during the presentation the audience asks the navigator to move to a specific position in the virtual world which is not part of the preconfigured tour. In this case, it is required that the stereoscopic eye distance is computed based on the new position in the virtual world. Therefore, both potential tour-related situations have to be covered by our approach.

\section{Stereoscopic configuration of CAVE's with a semi- immersive hybrid-dimensional monitor}

Nowadays, many different CAVE configurations exist, e.g. [1], [13], [14]. Here, we use the CAVE2 $2^{\mathrm{TM}}$ which is a circular display environment providing a $330^{\circ}$ panorama view [3], [13], [15]. With a diameter of $7.40 \mathrm{~m}$ it accommodates groups of up to 20 people, such as school classes, university seminars or company delegations. In its center, it provides space to communicate and to discuss the visualized environment from different perspectives, providing a direct combination of real and virtual world.

To configure CAVE tours, a device is required which is able to a) show the stereoscopic virtual map of the model visualized in the CAVE including a visual representation of the tour points to enable a precise orientation and navigation inside the virtual world, b) providing $2 \mathrm{D}$ visualization to show e.g. the sequential order of the tour and provide a GUI to change the settings of the tour and the stereo effect, and c) to be able to directly test the stereoscopic configuration.
For this purpose, a zSpace $200 \AA$, a semi-immersive 3D monitor is used. We developed a software for the zSpace which shows an overview map of the virtual world shown in the CAVE2 (following the world's in miniature - WIM - metaphor [16]). We call this approach Stereoscopic Space Map (or short in the following sections: Space Map). Different navigation methods were implemented to change the view in the virtual world by using the Space Map, partly based on our previous work [17].

A huge advantage of this approach is the fact that the tour and its stereoscopic optimization can be done using the zSpace before entering the CAVE2, because the use of this large-scale virtual environment is quite cost-intensive.

\section{Methods}

Since their invention, CAVEs are used for different purposes, but very frequently they are used for group presentations. Usually, only a single person or a very small group guides the tour through the virtual environment. Often, this is done by using wand-like devices. Alternatively, visual navigation interfaces providing access to additional information, e.g., tablet computers have been used for more than a decade in CAVEs [18]. But standard tablets are not able to stereoscopically visualize and interact with spatial structures in 3D space.

\section{Stereoscopic Space Map}

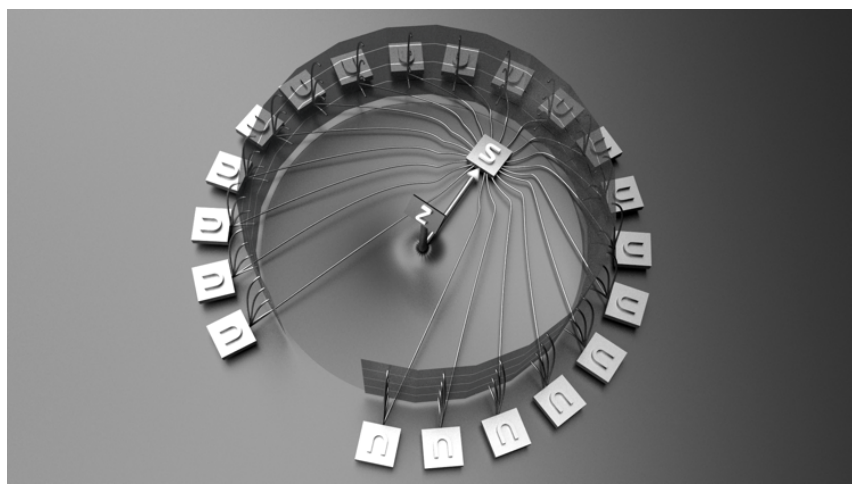

Figure 1. Stereoscopic Space Map - Hardware: This illustration shows the $3 D$ monitors (here: semi-transparent) of the CAVE2. Four of them are each connected to a single node $(\mathrm{n})$. The nodes are connected to a head node server (s). The zSpace system (z) communicate with the server

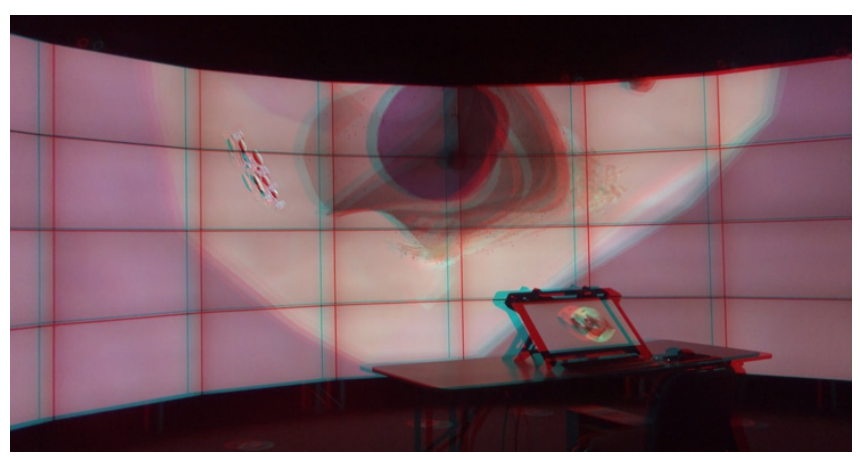

Figure 2. Stereoscopic Space Map - Hardware: Right bottom: the zSpace $200 \AA$ showing the map of the cell, in the background: the CAVE2 ${ }^{T M}$ environment, showing the nuclear region of the cell (red/cyan anaglyph stereo image) 
To enable pre-tour and on-tour stereoscopic effect configuration, we developed the Stereoscopic Space Map. On one hand, this map is used to provide the navigator with a preview of the stereoscopic setting, on the other hand it tries to calculate the best eye distance based on the distance to the closest object to the actual camera position (see section Stereoscopic 3D Methods).

During presentations in CAVE2, the navigator as well as the audience usually lack an overview of a complex environment. Of course, an overview like a map can be shown directly on the displays of the CAVE2 [19]. Especially CAVE2 is often used for hybrid-dimensional visualization and interaction, combining 2D and 3D approaches [17]. But this hybrid reality visualization will disturb the immersion as well as the 3D-stereoscopic effect, in case the CAVE2 should just represent the panorama window to the virtual world - and here, a 3D model should be explored (see also section Stereoscopic 3D Methods).

\section{Hardware}

The hardware used consists of a zSpace 200® [15, SOHD15], representing the navigator's display, whereas the virtual world is displayed by CAVE2 ${ }^{\mathrm{TM}}[15]$.

CAVE2 is a circular display environment consisting of 20 four-panel columns (with 46" 3D LCDs) providing a $330^{\circ}$ panorama view [13], [15] (Figure 1). With a diameter of $7.40 \mathrm{~m}$ and a height of $2.70 \mathrm{~m}$ it is appropriate for audiences with approx. 20 members.

Because the current navigation device, the wand (in our case a Sony PlayStation $3 \circledR$ controller connected to the CAVE2), should be replaced by the Space Map and a fluent and precise navigation should be enabled, high-resolution 3D interaction is required. The zSpace 200 is a passive Full HD 23" 3D monitor equipped with infrared-light-based head tracking system and a zStylus pen with three buttons and vibration capability for 3D interaction [20]. Another positive aspect is the fact that the zSpace is a quite mobile and compact device - therefore it takes approx. 10 minutes to place the zSpace system in the center of the CAVE2.

Both, the CAVE2 as well as the zSpace use circularly polarized glasses for creating the $3 \mathrm{D}$ effect, therefore the parallel use of both technologies is possible.

The zSpace system used in context of this work consists of the zSpace 200 monitor, an optional standard 2D monitor, plus a connected computer. Figure 1 shows how the zSpace system is connected to the CAVE2. Each monitor column is connected to one out of 20 node computers $(n)$ which are synchronized via a head node server $(s)$. The zSpace system $(z)$ communicates unidirectionally with the head node server. Figure 2 shows a photo of the zSpace/CAVE2 setup.

\section{Software}

The two displays are used in conjunction with two different software packages. The navigator software used for the Space Map is an extended version of the CELLmicrocosmos 1.2 CellExplorer (CmCX, available at http://Cm1.CELLmicrocosmos.org) [21], [22], whereas the high quality rendering engine supporting largescale visualization is Omegalib [19]. CmCX is a software which is used for educational as well scientific cell exploration and visualization [23]. In its context, a number of different cell models were generated - some of these models will be used for the experiments in the following sections.

The navigation actions are transferred from $\mathrm{CmCX}$ to Omegalib using a TCP/IP connection. By using $\mathrm{CmCX}$ as a navigation interface, the navigator is able to use a $3 \mathrm{D}$ overview map, including associated background information shown on a separate 2D monitor, plus an overview of the whole tour (Figure 3.1). In this way, the interactivity is maintained, while the camera in the virtual world moves in a movie-like fashion between different points of interest.

This approach is similar to the Worlds in Miniature (WIM) approach [16]. Here, the idea is to present a smaller simplified model of the virtual world to improve the navigation and interaction with the virtual environment. The initial approach integrated the WIM into the virtual environment - it was floating in front of the user while he was fully immersed in the virtual world. An advantage of our approach is the fact that the WIM does not block the view between the virtual world and the navigator, because it is placed in the center of the CAVE2.
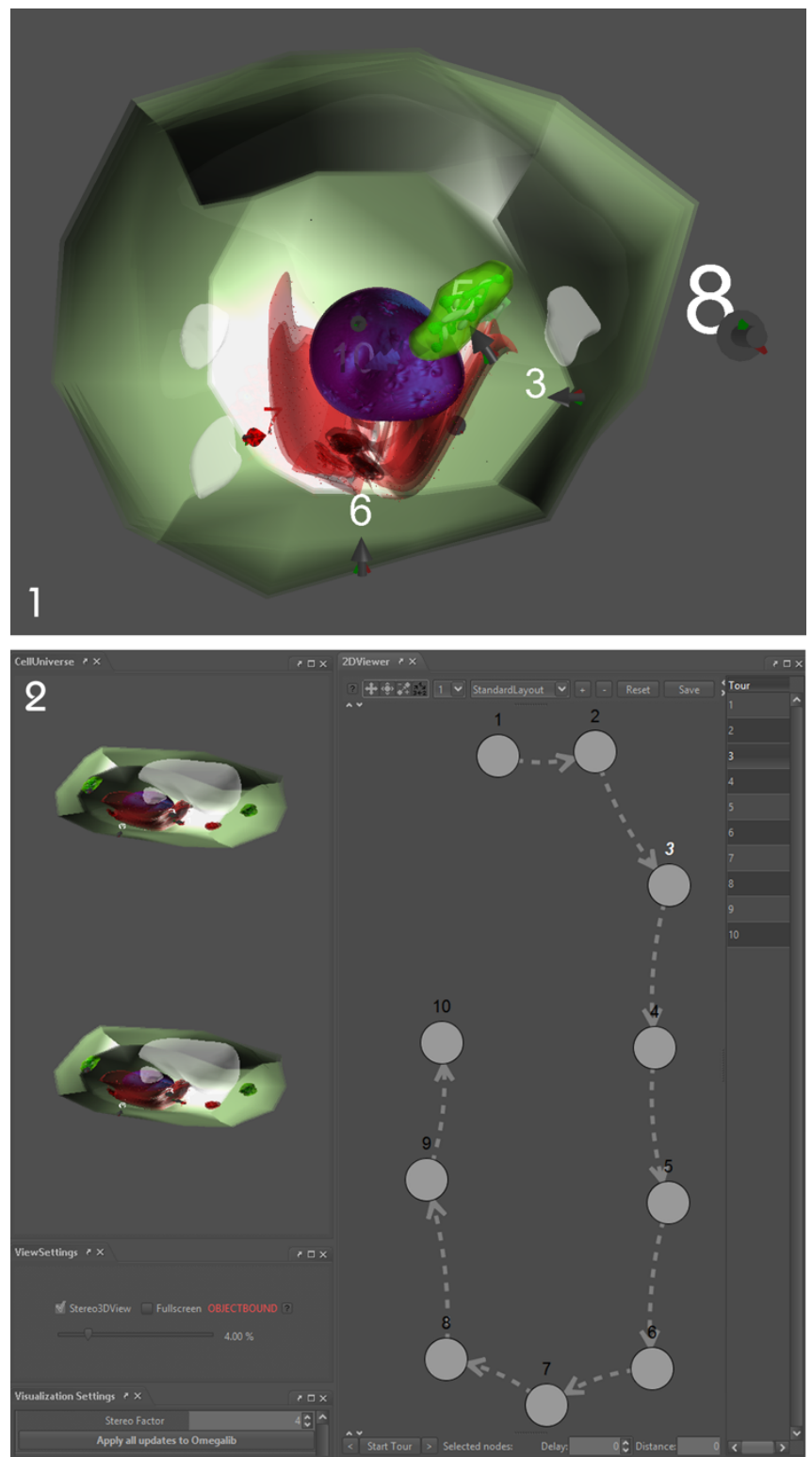

Figure 3 Stereoscopic Space Map - Software: 3.1: the plant cell as shown in Space Map in Full Screen mode; 3.2: the tour shown in the Stereoscopic Space Map: left top: the cell shown in top/bottom stereo mode, left bottom: the slider for the stereoscopic eye distance in the Space Map $\mathrm{S}_{\mathrm{S} 3 \mathrm{D}} \mathrm{Cmcx}_{\mathrm{m}}$, and below the slider for the stereoscopic eye distance in Omegalib $\mathrm{S}_{\mathrm{S}_{3} \mathrm{D}} \mathrm{OL}$, right: the tour and its sequential tour points 
The Space Map realized with the zSpace and $\mathrm{CmCX}$ provides different interaction techniques supporting six degrees of freedom (6DOF). Navigation using the zStylus pen was first introduced in our previous work discussing a hybrid-dimensional visualization and interaction approach by using the zSpace [17]. In Floating Mode it is possible to highlight each component of a model and in case nothing was selected - to freely move through the virtual map. By selecting an object and pressing the center button of the zStylus pen, the user changes from Floating Mode to Object-Bound Mode. In this mode, the user can rotate around selected components by vertically and horizontally moving the zStylus pen, and the distance towards the component can be changed by moving the zStylus pen forward or backward, respectively.

The position and orientation of the zStylus pen is mapped to a $3 \mathrm{D}$ pointer in the virtual map which can also be used to change the perspective in the virtual world shown in the CAVE2. For this purpose, the navigator can use the left zStylus pen button to transfer the actual view of the zSpace to CAVE2. Alternatively, clicking the right mouse button will transfer the orientation and position of the 3D mouse pointer to the CAVE's camera. The direction of the pointer reflects the direction of the camera. In this way, 6DOF camera positioning is possible.

\section{Stereoscopic 3D Methods}

It was previously mentioned that it is problematic to use CAVE2 with hybrid-dimensional visualization in case virtual worlds should be presented to an audience. The combination of a $2 \mathrm{D}$ interface with a $3 \mathrm{D}$ virtual world will be problematic because of potential pop-out effects associated with the virtual world: the $2 \mathrm{D}$ visualization area would be usually located in the projection plane (Figure 4.1, $A_{\text {projection }}$ ), but 3D objects in the virtual world would be often located between the projection plane and the near plane (Figure $4.1, A_{\text {near }}$ ), exceeding the 2D layer. Moreover, it would be also problematic to show the 3DS overview map in front of the virtual world, because the stereoscopic visualization would have to be adjusted for a) the virtual world in the background, as well as b) for the virtual map in the foreground. In this way, the virtual world should be restricted to the space between a) $A_{\text {projection }}$ and $A_{f a r}$, and the virtual map would have to be visualized between b) $A_{\text {projection }}$ and $A_{\text {near }}$ (Figure 4.1). In this way, pop-out effects for the virtual world would have to be heavily restricted or completely omitted - excessively limiting the representation of the virtual world.

3D-stereoscopic navigation requires special precautions, especially if large differences in scale have to be bridged. In our previous publication we introduced two different methods to optimize the stereoscopic vision.

Here, our previously discussed dynamic interactive projection plane method is used which is especially relevant in case large virtual cells are visualized, bridging the molecular and mesoscopic scale with differences of up to a factor of 100,000 [12].

Figure 4.2 illustrates the static interactive projection plane method. Here, the user navigates around the center of a single object. In our zSpace-based approach this is the case if the user moves around the center of the selected object by keeping the center zStylus button pressed while moving the zStylus pen. The eye distance decreases the closer the navigator moves towards the object.

Figure 4.3 depicts the dynamic interactive projection plane method. Because in this situation many objects of different size and location exist in the virtual world, the eye distance has to be adjusted based on the closest object to the user. Also here the eye distance decreases the closer the navigator moves towards the object, but the reference object is always the one in the center of the view.
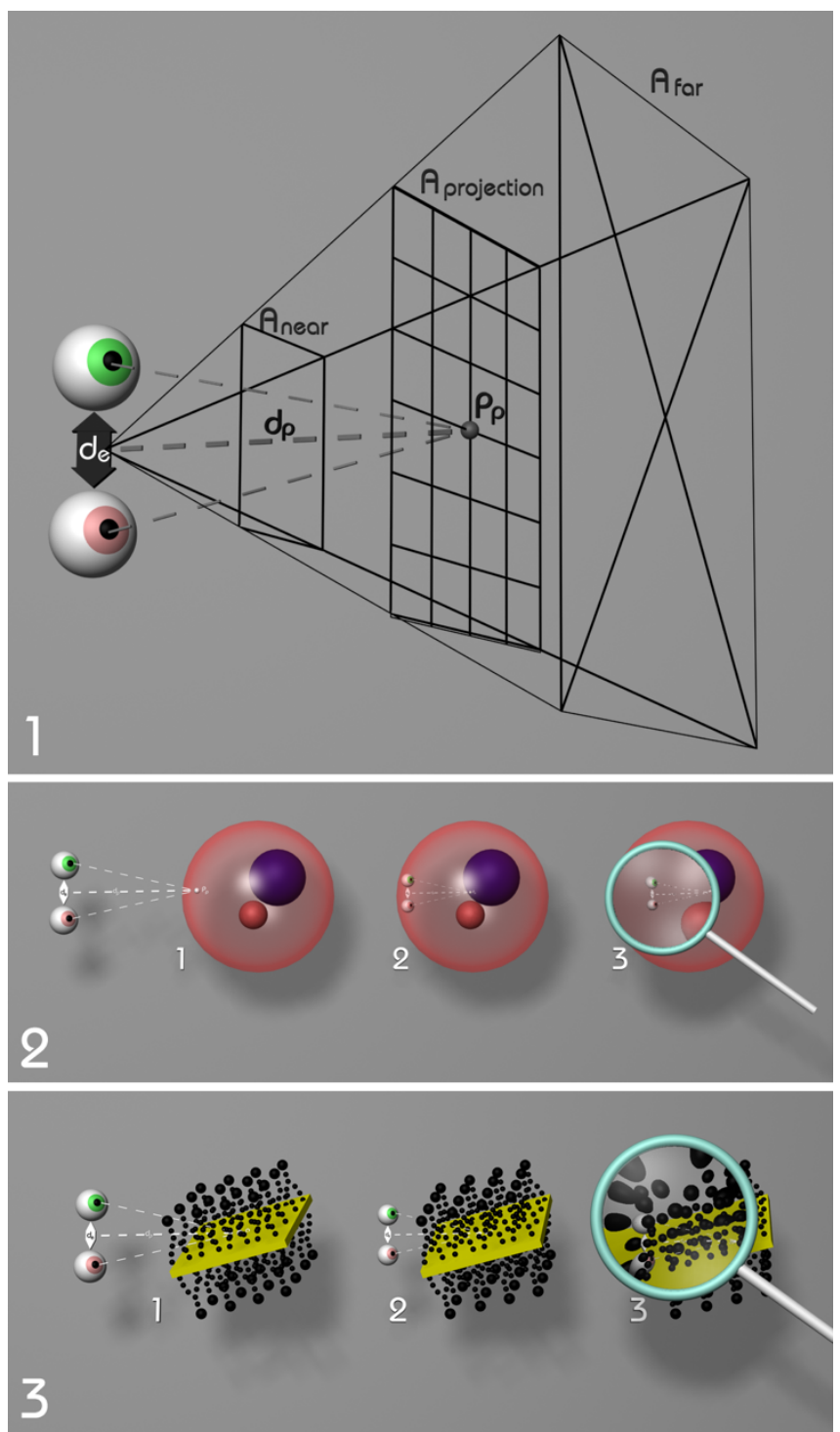

Figure 4: Automatic computation of the stereoscopic eye distance: 4.1: Correlation between eyes' distance and their distance to the picked point. $d_{e}$ : distance of eyes, $d_{p}$ : distance between eyes' center and $P_{p}, P_{p}$ : picked point, $A_{\text {projection: }}$ projection plane, $A_{\text {near }}$ : near plane, $A_{\text {far }}$ : far plane; 4.2: static interactive projection plane $S 3 D$ method; 4.3 dynamic interactive projection plane S3D method

The stereoscopic eye distance in the Space Map is defined as

$$
E_{\text {Dist_CmCX }}=\frac{\left(\frac{S_{S_{3} D_{-} C m C X}}{10}\right)^{2}}{\frac{1000}{d_{p}}}
$$

where $S_{S 3 D C m C X}$ is the slider for the stereoscopic eye distance shown in Figure 3.2 between [0..100]. In case of the dynamic interactive projection plane method, $d_{p}$ is the distance between the picked point $P p$ and the viewport's center position in the virtual world in Figure 4.1. In case the static interactive projection plane method, $d_{p}$ is the distance between the center of the picked object and the viewport's center position in the virtual world in Figure 4.3. The stereoscopic eye distance in Omegalib is computed as 


$$
E_{\text {Dist_omegalib }}=E_{\text {Dist_CmCX }} * S_{S 3 D_{-} O L}
$$

where $S_{S 3 D} O L$ is the value of the Omegalib slider in Figure 3.2.

Please note that the eyes shown in Figure 4.1 represent the virtual eyes of the navigator which means the position where the software predicts the eyes to be. Usually this position could be computed by using head tracking, but as previously mentioned head-tracking is counterproductive in this context. Therefore, the positon of the eyes is centered on the screen and it is assumed to be in a specific distance from the screen.

Our previous approach was extended here to create tours which provide the capability to save the stereoscopic setting at every tour point (Figure 3.2). For this purpose, the user can use the Space Map to navigate to a position which he later wants to present inside the CAVE, and create a tour point at this position, containing the orientation and position of the camera at this time point, as well as the computed eye distance based on the interactive projection plane method. A huge advantage of this a priori navigation is that the camera movement during on-tour presentation can be linearly interpolated, moving smoothly from one tour point to the next one.

The generation of the tour points is useful to define the stereoscopic settings during the pre-tour configuration. But it is also possible to pre-compute the stereoscopic eye distance during the on-tour navigation. In this way, the previously-mentioned 3D pointer can be used to pick a position in the 3D environment and directly transfer it to the CAVE2. Both approaches will be used in the following experiments.

Figure 3.2 shows now the two GUI elements which can be used to configure the stereoscopic eye distance. They will be used in the experiments and they have two modes. In the automatic stereoscopic mode, the value of the $\mathrm{CmCX}$ Stereoscopic Slider represents a factor which is used to scale the computed eye distance. Moreover, the resulting value is multiplied with the one of the Omegalib Stereoscopic Slider for the CAVE2. In the manual stereoscopic mode, the value of the CmCX Stereoscopic Slider as well as the one from Omegalib Stereoscopic Slider are independent from each other and are used to absolutely define the eye distance. In this way it is possible to optimize the stereoscopic effect to individual preferences, whereas the navigator always has to keep in mind that the stereoscopic effect has to be both, visually compelling and comfortable for the audience.

\section{Experiments}

To show that the automatic stereoscopic adjustment in combination with a virtual map and the virtual world is reasonable for group presentations from the navigator's perspective, an expert user study was conducted. Only participants with a lot of experience in group presentation in virtual environment were selected, resulting in a small group group of adequate participants, who were also not involved in this publication. Finally, eight participants (seven males, one female) with an average age of approx. 35 years were recruited. Each participant had experience with the usage of CAVE2 in combination with wand navigation for more than one hour, more precisely: three $1-10 \mathrm{~h}$, four $11-100 \mathrm{~h}$, one more than $100 \mathrm{~h}$. In addition, each of them had more than 11 hours experience with VR-related technology, such as CAVEs, HMDs, stereoscopic devices, etc.; more precisely, six 11-100 h, and two more than $100 \mathrm{~h}$. Two of them had minor experience with the zSpace. In this way, it was guaranteed that only users who have active experience with CAVEs and S3D visualization contribute to this study. Each of these eight persons participated in the role of a single navigator who was presented with preconfigured tours unknown to him.

Before the experiment started, the navigator was introduced to the usage of the zSpace and the usage of the zStylus pen in an independent scenario.

To evaluate the stereoscopic effect, six scenarios were designed by the authors of this work using the previously described methodology. Scenarios 1 to 3 used the new approach discussed in this publication combining CAVE2 and zSpace, scenarios 4 to 6 used more conventional techniques, excluding the zSpace from the experiment. Moreover, scenarios 1, 2, 4 and 5 represent tours based on pre-tour configurations, whereas 3 and 6 represent ontour presentations, where the stereoscopic effect has to be interactively optimized (because no tour points containing eye distance information exist). The six scenarios were based on three different cell models: two interpretative models of an animal cell and a plant cell which were modeled in context of the CELLmicrocosmos project, and one 3D-microscopy-based model of a neuron cell downloaded from the Cell-centered database (CCDB-ID 77503) [23], [24]. Each cell model contains a number of cell components, such as the nucleus, cell membrane, mitochondrion, etc.

Scenario 1) Linear Tour with zSpace: A linear tour was prepared by the authors containing tour points representing the camera position, camera orientation, as well as the stereo effect. The navigator has to explore this model by sequentially visiting the tour points using the forward/backward buttons on the keyboard. To be able to keep track of the whole virtual world during the navigation and to see, where the different tour points are located in 3D space, the zSpace is used as a virtual map (Figure 3.1).

Scenario 2) Tour Points in $3 D$ with zSpace: Again, a linear tour was prepared like in Scenario 1, but this time, the navigator had to directly click at the different tour points in 3D space (Figure 3.1). The tour nodes are represented by arrows, which indicate the position and orientation of the camera. This task requires some practice from the navigator, because tour points have to be clicked in 3D space by using the zStylus pen.

Scenario 3) Free Navigation with zStylus Pen and zSpace: Here no linear tour exists, but a number of cell components were indicated to be visited. For this purpose, the navigator can use the zStylus pen buttons to transfer the actual view of the zSpace or the position of the 3D pointer to CAVE2.

Scenario 4) Linear Tour: Like in Scenario 1, a tour was conducted, but this time, the zSpace cannot be used to visualize the virtual map. Here the navigator can therefore only use the CAVE2 for the visualization and, starting with this scenario, the zSpace was not used anymore.

Scenario 5) Tour Points in $2 D$ with Monitor: Like in Scenario 4 , the tour points were predefined. The navigator is provided with an overview of the tour as a network and he can click on the different tour points on an abstract 2D map shown on a standard 2D monitor (Figure 3.2 left), containing no additional information about the location of the different tour points, in contrast to Scenario 2 .

Scenario 6) Free Navigation with Wand: In this optional scenario, the navigator was able to use the wand to navigate through the environment. Because all participants had more than $1 \mathrm{~h}$ experience with the wand, this scenario was only practically used by one participant who wanted to refresh his wand navigation experience. To change the eye distance settings, a menu has to be toggled with the wand, providing a slider to change the eye distance.

Each linear tour and tour point scenario contained ten tour points based on 5 distance classes, as shown in Figure 5: 1) 30,000 
$\mathrm{nm}, 2)$ 10,000 nm (Figure 3.1: tour point 8), 3) 1,000 nm (Figure 3.1: tour point 6), 4) $100 \mathrm{~nm}$ (Figure 3.1: tour point 7), 5) $10 \mathrm{~nm}$. The distance classes are based on the computed $P_{P}$ in Figure 4 Therefore, distance differences of 3 orders of magnitude were evaluated.

During each of the scenarios, the navigator had to visit five subsequent tour points in automatic stereoscopic mode (using the interactive projection pane method), and five tour points in manual stereoscopic mode. In automatic stereoscopic mode, the navigator was limited defining the $\mathrm{zSpace}$ eye distance default value and the CAVE2 factor at the beginning of a tour. In manual stereoscopic mode, the navigator was allowed to change the absolute eye distance settings for each tour point for the zSpace as well as the CAVE2 at every tour point. For both purposes, the $\mathrm{CmCX}$ Stereoscopic Slider and the Omegalib Stereoscopic Slider are used. In this way, it is possible for the navigator to compare the conventional approach - the change of the eye distance by a slider - to the new approach, which automatically adjusts the eye distance.

The task for the navigator was at each step to find a stereoscopic eye distance setting which is good in terms of providing an appropriate $3 \mathrm{D}$ experience and being comfortable for the eyes of a potential audience. The duration of a single experiment was between approx. 60 to 90 minutes.

\section{Questionnaire}

The following questions were asked after the five to six experiments:

Q1. A general question: what is 3D Stereoscopy for you?

Q2. Please rate the quality of the automatically adjusted stereo effect in the zSpace.

Q3. Please rate the quality of the automatically adjusted stereo effect in the CAVE2.

Q4. How did the automatically adjusted stereo effect in the zSpace/CAVE2 worked in combination? Did the resulting eye distances match?

Q5. How did you feel about using two different stereoscopic devices in parallel?

Q6. Was it problematic to alternating focus the eyes on different stereoscopic displays?

Q7. Did you prefer the manual or automatically adjusted stereo setting? Do you prefer to adjust the stereo distance of the eyes manually or automatically, in terms of a) precision, b) speed, c) orientation, d) immersion, and e) attention? And what is your overall rating?

\section{Definitions and Scales}

Q1 was on the scale: excellent (numeric value: 1), good (2), okay (3), don't care (4), superfluous (5), annoying (6). Q2 to Q5 were on the scale: excellent (1), good (2), fair (3), problematic (4), poor (5). Q6 had the scale: no (1), small (2), somewhat (3), problematic (4), extremely problematic (5). Q7 had the answers automatic, manual, or both stereoscopic adjustment approaches.

The definitions for Q7 are the following:

- "Precision" refers to the accurateness to select a specific location and orientation in the virtual world.

- "Speed" refers to the required time to move from one position to the next one.

- "Orientation" refers to the sense of being aware of where one is localized in the virtual world (where is one's position, and the direction one is looking at, etc.).
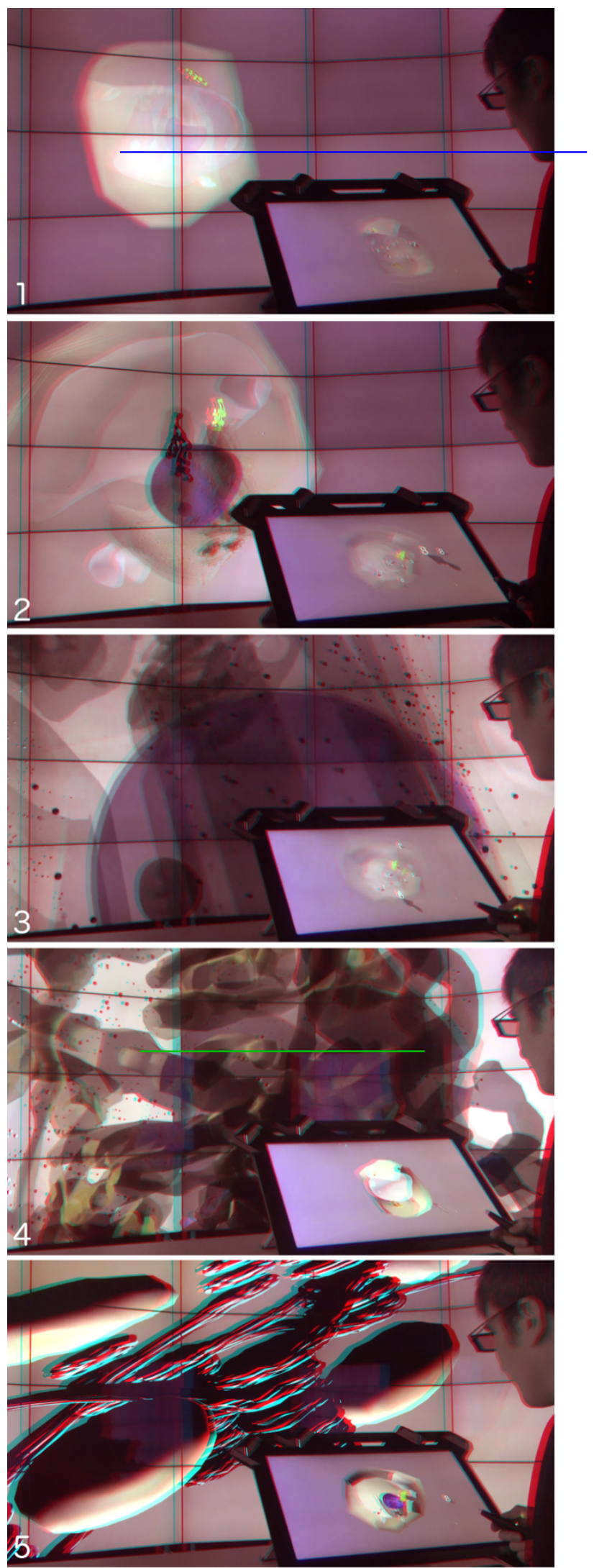

Figure 5. Visiting with the Space Map tour points representing 5 distance classes (red/cyan anaglyph stereo image) 
- "Immersion" refers to the sense of being present in the virtual world.

- "Attention" refers to the sense of being aware of the real world surrounding while navigating in the virtual world (i.e. in the role of a navigator, how aware are you of a potential audience, etc.).

Some of these questions were loosely inspired by the "igroup presence questionnaire (IPQ)" and the associated publication [25].

\section{Results}

The following section focuses now on the discussion and evaluation of the stereoscopic optimization for group presentations by using our Stereoscopic Space Map approach based on the navigator's perspective.

\section{Evaluation}

The evaluation showed that our proposed method leads already to good results. First, we wanted to know the attitude of the participants toward 3D Stereoscopy. Figure 6 shows that the overall attitude was quite positive which is no surprise in context of a CAVE expert study. The Y-axis shows always the number of participants. The value of 1.875 is even a little bit better than in our previous non-expert study in 2014 with 2.05 [12].

The best rating of the following questions was achieved by Q2 (Figure 7, Blue): the quality of the automatically adjusted stereo effect in the zSpace was rated with 2.0. Obviously, the approach based on the interactive projection plane method is quite advanced and was appropriately ported to the zSpace. With 2.25 the automatically adjusted stereo effect/Q3 in the CAVE2 also works quite well (Figure 7, Red). The results of Q4 with 2.75 show that the eye distances between zSpace and CAVE2 worked fairly well. Therefore, the approach is promising, but there is room for improvements.

In the preliminary discussions among the authors concerning the experiments, the question was asked if it might be uncomfortable to use two stereoscopic devices in parallel. Based on Q5 (Figure 7, Orange) and Q6 this question can now be answered: The usage of these two displays in parallel works quite well with a rating of 2.375 and also the eye refocusing during the parallel usage was usually no problem for the participants, with an average rating of 2.625 (Figure 8). But looking at the graphs of Q5 and Q6 it is obvious that one participant had immense problems by using two displays (and both negative ratings are based on the same participant). It has to be said that the experiments where conducted in a way that the need for eye refocusing was quite demanding: the stereo effect of the tour points had often to be adjusted, especially because the automatic adjustment (which would be the regular application case for this approach) was combined with the manual adjustment (for evaluation purposes). Moreover, the duration of an experiment with up to 90 minutes was quite long. Therefore, the scenarios do not represent a presentation session, but the preparation of such a session, during which the stereo effect is optimized for every single tour point. It is not a surprise that this demanding task is not compatible with all participants. In our previous study in 2014 there was also one participant who had to stop the experiment because he was not able to perceive stereoscopic images without motion sickness [12]. Without this outlier, both results would be good, with Q5 2.29 and Q6 2.0.

The results of the final question Q7 in Figure 9 reveal now how the automatically adjusted stereo effect compares to the manually adjusted one. First of all, the precision was rated better than expected, because it could be expected that the precision of the manually adjusted stereo effect is better than the computed one.

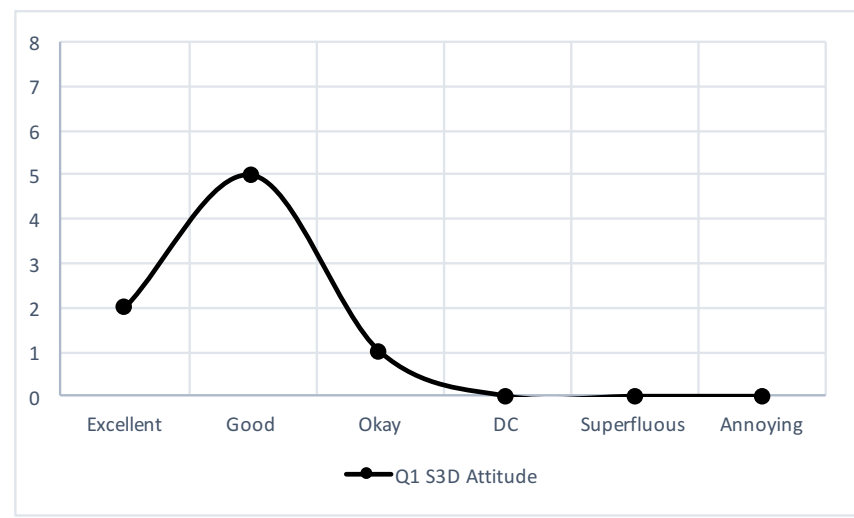

Figure 6. Results Q1: What is 3D Stereoscopy for you?

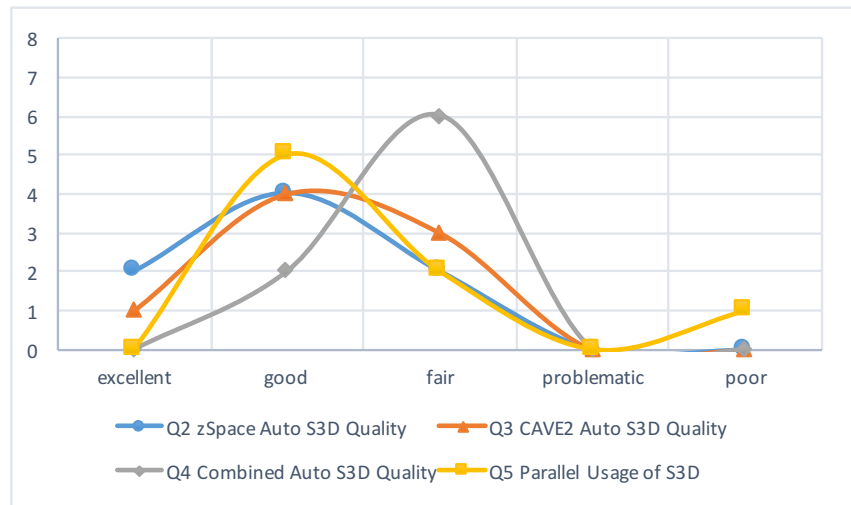

Figure 7. Results of Q2 to Q5: Blue - Results Q2: Quality of the automatically adjusted stereo effect in zSpace; Red - Results Q3: Quality of the automatically adjusted stereo effect in CAVE2; Grey - Results Q4: Quality of the automatically adjusted stereo effect in combination zSpace/CAVE2;

Orange - Results Q5: Parallel use of two stereoscopic devices

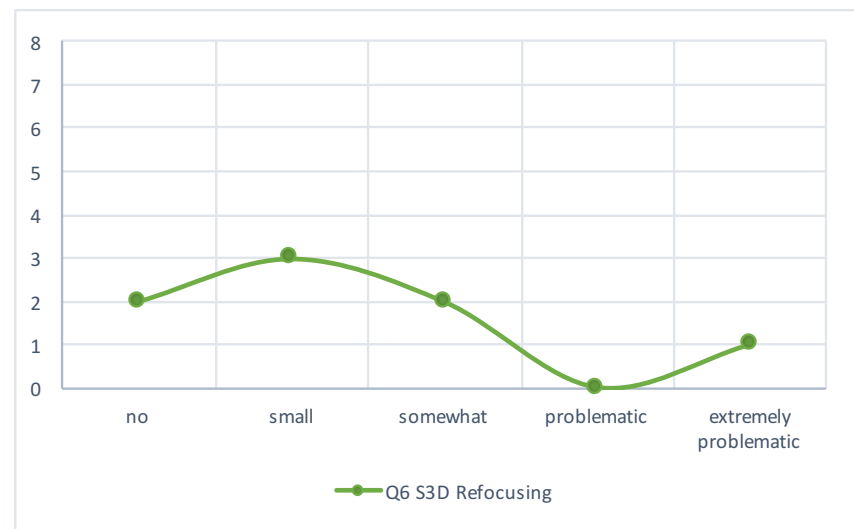

Figure 8. Results Q6: Problems during eye refocusing when using two stereoscopic devices (CAVE2/zSpace)

Only three participants preferred the manual setting, five of them liked both or even preferred the automatic setting (two participants). It is no surprise that in terms of speed the automatic adjustment was preferred by far, and only a single person was also okay with the speed during the manual setting. The results for the 
orientation shows that $50 \%$ preferred the automatically adjusted effect, and $50 \%$ were fine with both. Interestingly, one person preferred the manual setting in terms of immersion. Again, the largest part of participant favored the automatic setting and three were fine with both approaches. Also, the attention is negatively impacted by manual setting, only one person was okay with both, but all others clearly preferred the automatic one. The overall rating shows that five people preferred the automatic setting, and only two persons were satisfied with both approaches. Based on the previous ratings for Q7 it can be predicted that the only reason might be that three people clearly preferred the manual setting in terms of precision.

A reason that the precision seems not to be perfect for the automatic setting is that the automatically defined values were directly assigned to the tour, basically without optimizing the values afterwards. Although the proposed concept worked quite well - the pre-configuration of the stereoscopic settings in the virtual map represented by the zSpace and then using these settings during the presentation in the virtual world of the CAVE2 optimal values can only be achieved if the stereoscopic setting is reevaluated for each tour point in the CAVE2 before presentation. Whereas the algorithm only takes the nearest object in the center of the viewport (of the zSpace) into account, the navigator is able to judge the eye distance settings based on all objects in the viewport.

Moreover, as the CAVE2 has a circular shape, it might happen that objects outside the zSpace's field of view might be closer to the navigator inside the CAVE2. In this case, the computed stereo effect might be too strong. Especially for this purpose the stereoscopic setting should be optimized in the CAVE2 before showing a tour to an audience. Potential future work would be to compute the eye distance based on all closest objects in the environment.

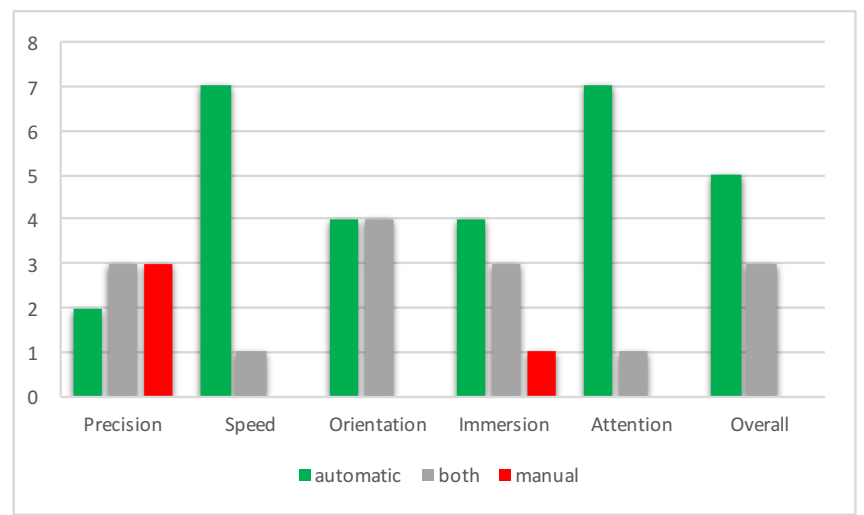

Figure 9. Results Q7: Preference automatically vs. manually adjusted stereo effect in terms of different VR-related aspects

\section{Conclusion}

This work introduced an alternative to - what we think to be a basic misconception in CAVE presentations. Usually, the navigator is head-tracked to provide him with the optimal perspective and stereoscopic visualization in the CAVE. But as the presentation is not intended to provide an optimal experience to the navigator, but to the audience, we developed a new approach to configure the camera positions and the stereoscopic effect pre-tour as well as on-tour. For this purpose, $\mathrm{CmCX}$ was used in conjunction with a semi-immersive stereoscopic monitor, the zSpace. The zSpace was used for the navigation and the configuration of the stereoscopic effect in CAVE2.

The previously published interactive projection plane approach was used to pre-compute the stereoscopic effect in the CAVE2. To show that this approach leads to good results, an expert user study was conducted, comparing the conventional manual adjustment of the eye distance with the automatic one. Only those people participated who had already previous experiences with using the CAVE2 for presentation purposes in combination with a wand-like device.

To test the quality of our proposed method in the context of different navigation modes, six different navigation scenarios were used, combining new and more conventional navigation techniques in CAVEs with the navigation through 3D models of cells. Four of these scenarios contained tour points with five different distance classes with a variation of distance differences of 3 orders of magnitude (measured from the center of the projection plane in the zSpace to the closest object in the view's center). Two additional scenarios allowed the free navigation through the virtual world.

Seven of eight participants were convinced by the automatically adjusted stereoscopic effect. On a scale of 1 (optimum) to 5 (worst), the automatically adjusted stereo effect for the zSpace achieved 2, the CAVE2 achieved 2.25, and the combination of both effects achieved - representing the subjective matching of eye distances - 2.75 over all eight participants. Whereas seven participants experienced no problems with the parallel usage, one participant experienced typical motion sickness symptoms because of the visually demanding task to reconfigure the stereoscopic settings over approx. 75 minutes. The overall rating of the usage of two different stereoscopic devices in parallel was 2.625 , and refocusing the eyes 2.375 .

Comparing the automatic adjustment with the conventional manual setting by just using a slider to change the eye distance for every tour point, the automatic adjustment clearly outperformed the manual one in terms of speed and attention. Also, the preference in terms of orientation and immersion clearly showed a trend towards the automatic adjustment. Only in terms of precision the manual adjustment still showed a very small advantage for all participants. The overall rating showed that no participant preferred the manual setting, and 5 participants preferred the automatic one.

Although the ratings of the parallel usage are quite convincing, we proposed potential future improvements to our approach. On one hand, the setting of the stereoscopically predefined tours was fully based on the interactive projection plane method. The computation of the interactive projection plane method is only based on the closest object to the viewport's center in the zSpace, ignoring potentially closer objects in the largest part of the $330^{\circ}$ panorama view of the CAVE. However, keeping this in mind it is obviously already possible to achieve good results, as the navigator will usually choose the object in the center of the viewport as the closest point. Moreover, it is of course possible to reevaluate the position of the camera by inspecting the arrow in the virtual map of the zSpace. Therefore, the stereoscopic setting for every tour point could be optimized by the navigator before the tour starts.

This navigator-centric study of the Stereoscopic Space Map was focused on the value of the stereoscopic tour generation for the navigator. In parallel we started to evaluate the different new navigation modes of this approach. These results will be discussed in a future publication in combination with an audience-centric study to evaluate if the audience prefers common navigation techniques or the newly developed ones. 


\section{Abbreviations}

- $2 \mathrm{D}$

two-dimensional

- $3 \mathrm{D}$

three-dimensional

- 6DOF six Degrees Of Freedom

- CAVE CAVE Automatic Virtual Environment

- CmCX CELLmicrocosmos 1.2 CellExplorer

- HMD Head-Mounted Display

- S3D Stereoscopic three-dimensional

\section{Acknowledgement}

We would like to thank all people who participated in the expert review.

\section{References}

[1] C. Cruz-Neira, D. J. Sandin, T. A. DeFanti, R. V. Kenyon, and J. C. Hart, "The CAVE: audio visual experience automatic virtual environment," Commun. ACM, vol. 35, no. 6, pp. 64-72, 1992.

[2] I. E. Sutherland, "A head-mounted three dimensional display," in Proceedings of the December 9-11, 1968, fall joint computer conference, part I, 1968, pp. 757-764.

[3] C. J. Fluke and D. G. Barnes, "The Ultimate Display,” in Proceedings of Astronomical Data Analysis Software and Systems (ADASS) XXV, Sydney, in press, 2016.

[4] S. Davis, K. Nesbitt, and E. Nalivaiko, "A Systematic Review of Cybersickness," in Proceedings of the 2014 Conference on Interactive Entertainment, 2014, pp. 1-9.

[5] J. T. Reason, "Motion sickness adaptation: a neural mismatch model.," J. R. Soc. Med., vol. 71, no. 11, p. 819, 1978.

[6] T. Kawai, M. Hirahara, Y. Tomiyama, D. Atsuta, and J. Häkkinen, "Disparity analysis of 3D movies and emotional representations," in Stereoscopic Displays and Applications XXIV, 2013, p. 86480Z86480Z-9.

[7] F. Speranza, W. J. Tam, R. Renaud, and N. Hur, "Effect of disparity and motion on visual comfort of stereoscopic images," in Stereoscopic Displays and Virtual Reality Systems XIII, 2006, p. 60550B-60550B-10.

[8] J. P. Wann, S. Rushton, and M. Mon-Williams, "Natural problems for stereoscopic depth perception in virtual environments," Vision Res., vol. 35, no. 19, pp. 2731-2736, 1995.

[9] A. Voronov, D. Vatolin, D. Sumin, V. Napadovsky, and A. Borisov, "Methodology for stereoscopic motion-picture quality assessment," in Stereoscopic Displays and Applications XXIV, 2013, pp. 864810-864810-14.

[10] A. Bokov, D. Vatolin, A. Zachesov, A. Belous, and M. Erofeev, "Automatic detection of artifacts in converted S3D video," in IS\&T/SPIE Electronic Imaging, 2014, pp. 901112-901112-14.

[11] T. Keip, Stereoskopie und 3D-Filme - Workflow Optimierungen im Konsum Bereich. Saarbrücken: VDM Verlag Dr. Müller \& Co. KG, 2010.

[12] B. Sommer, C. Bender, T. Hoppe, C. Gamroth, and L. Jelonek, "Stereoscopic cell visualization: from mesoscopic to molecular scale," J. Electron. Imaging, vol. 23, no. 1, pp. 011007-1 011007-10, 2014.
[13] A. Febretti, A. Nishimoto, T. Thigpen, J. Talandis, L. Long, J. D. Pirtle, T. Peterka, A. Verlo, M. Brown, and D. Plepys, "CAVE2: a hybrid reality environment for immersive simulation and information analysis," in IS\&T/SPIE Electronic Imaging, 2013, pp. 864903-864903-12.

[14] T. A. DeFanti, G. Dawe, D. J. Sandin, J. P. Schulze, P. Otto, J. Girado, F. Kuester, L. Smarr, and R. Rao, "The StarCAVE, a thirdgeneration CAVE and virtual reality OptIPortal," Future Gener. Comput. Syst., vol. 25, no. 2, pp. 169-178, 2009.

[15] W. J. Goscinski, P. McIntosh, U. Felzmann, A. Maksimenko, C. J. Hall, T. Gureyev, D. Thompson, A. Janke, G. Galloway, and N. E. Killeen, "The multi-modal Australian ScienceS Imaging and Visualization Environment (MASSIVE) high performance computing infrastructure: applications in neuroscience and neuroinformatics research," Front. Neuroinformatics, vol. 8, 2014.

[16] R. Stoakley, M. J. Conway, and R. Pausch, "Virtual reality on a WIM: interactive worlds in miniature," in Proceedings of the SIGCHI conference on Human factors in computing systems, 1995, pp. 265-272.

[17] B. Sommer, S. J. Wang, L. Xu, M. Chen, and F. Schreiber, "Hybrid-Dimensional Visualization and Interaction - Integrating 2D and 3D Visualization with Semi-Immersive Navigation Techniques," in Big Data Visual Analytics (BDVA), 2015, 2015, pp. $1-8$.

[18] Y. Yang, L. Engin, E. S. Wurtele, C. Cruz-Neira, and J. A. Dickerson, "Integration of metabolic networks and gene expression in virtual reality," Bioinformatics, vol. 21, no. 18, p. 3645, 2005.

[19] A. Febretti, A. Nishimoto, V. Mateevitsi, L. Renambot, A. Johnson, and J. Leigh, "Omegalib: A multi-view application framework for hybrid reality display environments," in Virtual Reality (VR), 2014 iEEE, 2014, pp. 9-14.

[20] “zSpace Product,” 2015. [Online]. Available: http://zspace.com/product. [Accessed: 02-Jun-2015].

[21] B. Sommer, J. Künsemöller, N. Sand, A. Husemann, M. Rumming, and B. Kormeier, "CELLmicrocosmos 4.1: an interactive approach to integrating spatially localized metabolic networks into a virtual 3D cell environment," in Proceedings of BIOSTEC Bioinformatics), 2010, pp. 90-95.

[22] B. Sommer, B. Kormeier, P. S. Demenkov, P. Arrigo, K. Hippe, Ö. Ates, A. V. Kochetov, V. A. Ivanisenko, N. A. Kolchanov, and R. Hofestädt, "Subcellular Localization Charts: A new visual methodology for the semi-automatic localization of protein-related data sets," J. Bioinform. Comput. Biol., vol. 11, no. 1, p. 1340005, 2013.

[23] B. Sommer, "CELLmicrocosmos-Integrative cell modeling at the molecular, mesoscopic and functional level," Doctorate Thesis, Bielefeld University, Bielefeld, Germany, 2012.

[24] M. E. Martone, A. Gupta, M. Wong, X. Qian, G. Sosinsky, B. Ludäscher, and M. H. Ellisman, "A cell-centered database for electron tomographic data," J. Struct. Biol., vol. 138, no. 1-2, pp. 145-155, 2002.

[25] T. W. Schubert, F. Friedmann, and H. T. Regenbrecht, "Decomposing the sense of presence: Factor analytic insights," in 2nd international workshop on presence, 1999, pp. 6-7. 